\title{
Dynamic Wetting Behavior of Vibrated Droplets on a Micropillared Surface
}

\author{
Zhi-hai Jia, Wei Lei, Hui-nan Yang, and Gang Wang \\ School of Energy and Power Engineering, University of Shanghai for Science and Technology, Shanghai 200093, China \\ Correspondence should be addressed to Zhi-hai Jia; zhhjia@usst.edu.cn
}

Received 25 September 2015; Revised 17 November 2015; Accepted 3 January 2016

Academic Editor: José L. Ocaña

Copyright (C) 2016 Zhi-hai Jia et al. This is an open access article distributed under the Creative Commons Attribution License, which permits unrestricted use, distribution, and reproduction in any medium, provided the original work is properly cited.

\begin{abstract}
The dynamical wetting behavior has been observed under vertical vibration of a water droplet placed on a micropillared surface. The wetting transition takes place under the different processes. In compression process, the droplet is transited from Cassie state to Wenzel state. The droplet undergoes a Wenzel-Cassie wetting transition in restoring process and the droplet bounces off from the surface in bouncing process. Meanwhile, the wetting and dewetting models during vibration are proposed. The wetting transition is confirmed by the model calculation. This study has potential to be used to control the wetting state.
\end{abstract}

\section{Introduction}

With the development of micro- and nanotechnology, superhydrophobic surface with a high static contact angle (CA) above $150^{\circ}$ and low contact angle hysteresis plays an important role in technical applications, such as drag reduction in microfluidic devices and dropwise condensation [1-3]. In particular, achieving a high CA of these superhydrophobic surfaces is not crucial; the behavior of the droplet moving on these surfaces and the velocity of the liquid leaving from these surfaces are important in most of the applications. And superhydrophobic surfaces are not always water repellent, such as condensation $[4,5]$. Therefore, restoring the characteristics of the superhydrophobic surfaces has gained widespread attention $[6,7]$.

In the previous research, theoretical models for liquid droplets wetting on rough and chemically inhomogeneous surfaces have been investigated [8-11]. Wetting of these surfaces is governed by the Wenzel model, where the liquid wets both peaks and valleys of rough surfaces, as shown in Figure 1(a). An apparent contact angle $\theta_{w}$ of a liquid droplet on a rough surface is given by the classical Wenzel equation

$$
\cos \theta_{w}=r_{w} \cos \theta_{E},
$$

where $r_{w}$ is roughness factor, defined as the ratio of the actual area of a rough surface to the geometric projected area. This factor is always larger than unity. And $\theta_{E}$ is an equilibrium contact angle of a liquid droplet on a corresponding flat surface.

Chemically heterogeneous surface is characterized by the apparent CA, predicted by the Cassie-Baxter wetting model, as shown in Figure $1(\mathrm{~b})$. The apparent $\mathrm{CA} \theta_{\mathrm{CB}}$ is defined by the Cassie-Baxter equation

$$
\cos \theta_{\mathrm{CB}}=f \cos \theta_{\mathrm{E}}+f-1,
$$

where $f$ is the fraction of the projected area of the solid surface that is wetted by the liquid. Although these two wellestablished theoretical models, Wenzel model and Cassie model, are frequently employed in demonstrating different wetting phenomena, the wetting mechanisms on the rough surface are not fully investigated yet, especially in the transition region between the Wenzel and Cassie states.

Recently, wetting transitions have been studied in a series of experimental and theoretical works [12-16]. It is shown that the wetting transitions are achieved by external stimuli, such as pressure $[17,18]$, vibration $[19,20]$, electric voltage $[21,22]$, evaporation or heat $[23,24]$, thermal chemical methods [25], solvent method [26], and magnetism [27]. Jung and Bhushan [12] found that the inertia force imposed on the droplet by vibration can overcome the adhesion force between the droplet and the rough surfaces; the droplet can bounce off from the surface and transit from Wenzel state to Cassie 


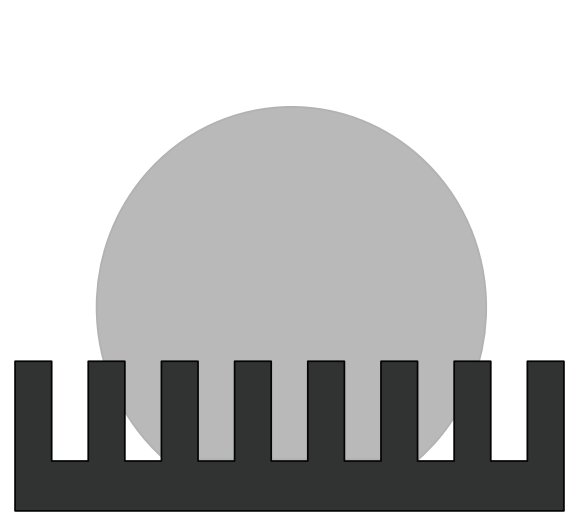

(a) Wenzel state

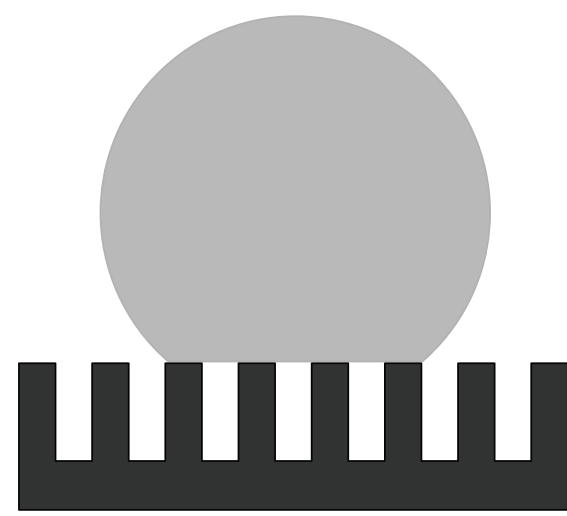

(b) Cassie state

FIGURE 1: Wetting state of rough surfaces.

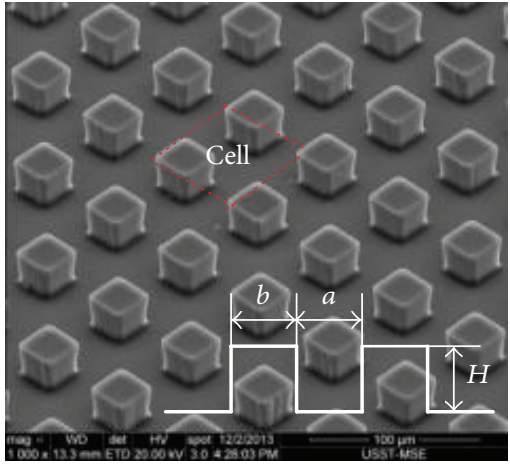

(a)

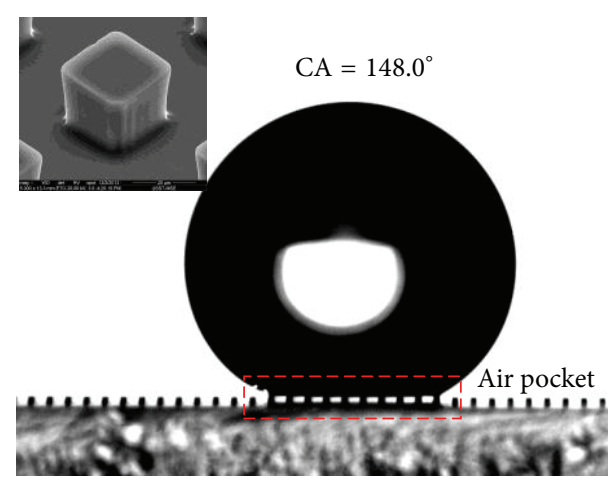

(b)

FIGURE 2: (a) SEM image of pillar structures made of PDMS ( $a$ is the pillar spacing, $H$ is the height of the pillars, and $b$ is the width). (b) Wetting state and contact angle of droplet of $4 \mu \mathrm{L}$ on the microstructured surface.

state. Bormashenko et al. [28] showed that the dynamic Cassie-Wenzel transition under vibration is more likely a $1 \mathrm{D}$ than a 2D affair. Meanwhile, the oscillations of droplets were widely studied on the hydrophobic or superhydrophobic surfaces [29-31]. However, most of them were focused on drop vibration models, and few experimental studies have been reported on the dynamic wetting behavior of vibrated droplets on patterned surfaces.

In this paper, dynamic properties (such as the droplet contact diameter, the CA, and the liquid height) of a vibrated droplet on patterned surfaces at resonance frequency are studied. The influence of vibration on the dynamic wetting states of a water droplet is investigated. And the wetting and dewetting models during the vibration are presented.

\section{Experimental Section}

2.1. Fabrication of Polydimethylsiloxane (PDMS) Rough Surfaces. Microstructured surfaces are fabricated using PDMS material by soft lithography, with pillars of $30 \mu \mathrm{m}$ in width, $30 \mu \mathrm{m}$ in height, and $30 \mu \mathrm{m}$ in pitch value. PDMS stamps were prepared in a replication molding process with a 6inch silicon wafer, which was covered with one layer of microstructured photoresist as a master. For preparation of the master, a layer $(30 \mu \mathrm{m}$ thickness ) of photoresist (SU-8, formulation 25, and a new negative near-UV thick photoresist) is spin-coated on a hydrophilic silicon wafer. Subsequently, the photoresist is patterned with the desired features, utilizing a printed foil mask. The polymer is allowed to cure for one hour at $90^{\circ} \mathrm{C}$ and to be cooled to room temperature before being removed from the master.

The surface morphology of the pillars, which is observed with a scanning electron microscope (SEM, QUANTA FEG 450 ), is shown in Figure 2(a). Deionized water is used as the liquid and the volume of each droplet is kept at $4 \mu \mathrm{L}$. Droplets are gently deposited on the substrate using a CA meter for the static CA. The CA value is $148.5^{\circ} \pm 2.5^{\circ}$, which is the average value of ten independent points, so the fabricated surface is a superhydrophobic surface [32]. As shown in Figure 2(b), the water droplet penetration into the space between the pillars is relatively small and it could be easily seen that the initial wetting state for PDMS surface is the Cassie state. Wetting of rough surfaces is characterized by the apparent contact angle; the apparent CA value is $113.7^{\circ}$ for PDMS surface in this work. According to the Wenzel and Cassie equations (1) and (2), the local apparent contact angles are $143.5^{\circ}$ and $148.3^{\circ}$, 


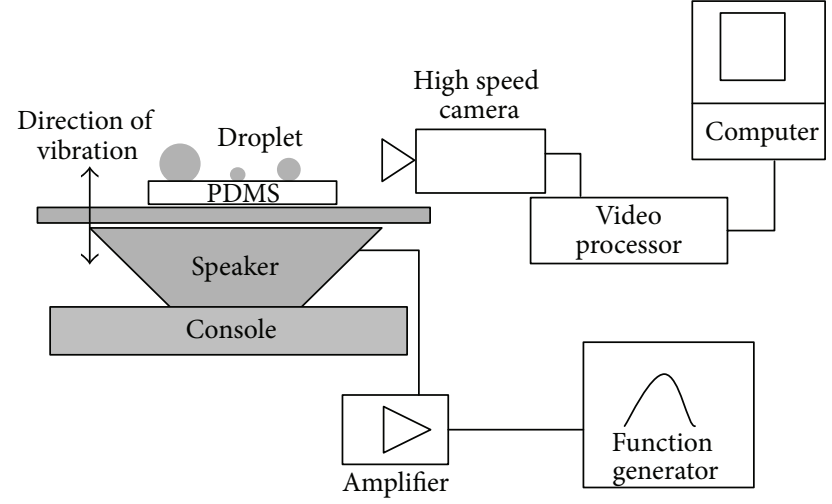

Figure 3: Experimental setup.

respectively. Thus, the experimental results are in accordance with the theoretical analysis: the initial wetting state is the Cassie state here.

2.2. Experimental Apparatus and Procedure. The experimental setup is shown in Figure 3. The droplet is rested on a microstructured surface and the surface is vertically vibrated with a loudspeaker (maximum peak-to-peak displacement $2 \mathrm{~mm}$ ). It is linked to a power amplifier connected to a digital function generator. The frequency and amplitude of a sinusoidal wave from a function generator can be adjusted. The vibration frequency was controlled between 0 and $250 \mathrm{~Hz}$ for measurement of the resonance frequency of a droplet. The process of the dynamic behavior was obtained with a high speed camera (OLYMPUS i-SPEED 3) operated at $2000 \mathrm{frames} / \mathrm{s}$ for each experimental run, and the dynamic properties of the droplets were then determined as a function of time. All experiments were carried out at $20 \pm 1^{\circ} \mathrm{C}$ and 50 $\pm 5 \%$ relative humidity $(\mathrm{RH})$.

\section{Results and Discussion}

In this work, the droplet is exposed to a vertical vibration. The amplitude increases during the experiment until the droplet first bounces off from the solid surface. The droplet oscillation gets vigorous with its shape and the triple line is moving. Figure 4 shows the images of droplets from the pillars PDMS surface at external forcing vibration frequency of $70 \mathrm{~Hz}$ and vibration amplitude of $0.31 \mathrm{~mm}$.

It reveals that the instantaneous dynamic characteristics of the vibrated droplets, such as the droplet contact diameter $D(t)$, CA $\theta(t)$, and the droplet height $H(t)$, are different at various time points. Meanwhile, the input waveform is sinusoidal signal, and the amplitude $A$ is a function of time $t, A(t)=A_{0} \sin (2 \pi f t)$, where $f$ is the frequency and $A_{0}$ is the half of the peak-to-peak amplitude of vibration.

Figure 5 shows the transient evolution of the droplet and amplitude $A(t)$ prior to the droplet bouncing-off. In one hand, $H(t), D(t)$, and $\theta(t)$ increase or decrease with the speaker vibration amplitude $(A)$, indicating that the droplet motion and external forcing are harmonious. It is in a resonance mode, which had been discussed in detail in our recent work [33]. Meanwhile, because of fluid elastic deformation, there is a time interval $\Delta t$ between the amplitude and the instantaneous dynamic characteristics of vibrated droplets, as shown in Figure 5(a). On the other hand, with the amplitude increasing and decreasing, the dynamic behaviors of a droplet can generally be classified into three different categories: compression process, restoring process, and bouncing process.

The compression process corresponds to the case where the vibrated droplet is continuously compressed as the top portion of the droplet is moving at a lower velocity in the positive $y$-direction than the bottom portion of the droplet. This behavior results in considerable decreasing of the liquid height during its vibration in the $y$-direction, as shown in Figures 4(a)-4(c) and 5(a). Also, the contact diameter between the droplet and the substrate is increasing (see Figure 5(b)) and the CA is decreasing (see Figure 5(c)). Such a situation occurs because of the partial filling of pillars with water and that results in a wetting transition. Generally, there are two main scenarios of wetting transitions, $1 \mathrm{D}$ and $2 \mathrm{D}$, as shown in Figure 6 [34]. In this case, as shown in Figure 6, the triple line and contact angle are continuously slowly changing and the triple line is not pinned. Thus, the dynamic CassieWenzel transition is more likely a $1 \mathrm{D}$ than a $2 \mathrm{D}$ affair. Also, it has been established that the transition occurs under the constant force per unit length of the triple contact line. It is not necessary to fill all surface intrusions but only those adjacent to the triple line [28].

On the rough substrate manufactured from strongly hydrophobic material, the Cassie wetting state corresponds to a higher free energy of the droplet/substrate system than the Wenzel wetting state. Vibrations provide the droplet with the necessary energy for penetration into pillars on the surface. The energy required when one "cell" is wetting is

$$
\Delta E_{\text {cell }}=4 b h\left(\gamma_{\mathrm{SL}}-\gamma_{\mathrm{SA}}\right)=-4 b h \gamma_{\mathrm{LA}} \cos \theta_{E},
$$

where $\gamma_{\mathrm{LA}}, \gamma_{\mathrm{SL}}$, and $\gamma_{\mathrm{SA}}$ are surface tensions on the liquidair, solid-liquid, and solid-air interfaces, respectively. $\theta_{E}$ is the Young contact angle of a flat surface. The number of such cells depends on the scenarios of wetting transition. As mentioned above, the transition corresponds to the $1 \mathrm{D}$ scenario of wetting transitions. Figure 7 shows the wetting behavior models. As it is shown in Figure 6, the number of "cells" is

$$
N=\frac{\left(\pi R_{\max }^{2}-\pi R_{0}^{2}\right)}{(a+b)^{2}}
$$

Thus, the necessary energy for wetting the surface in this process is

$$
E=N \Delta E_{\text {cell }}=\frac{-4 b h \gamma_{\mathrm{LA}} \pi\left(R_{\mathrm{max}}^{2}-R_{0}^{2}\right) \cos \theta_{E}}{(a+b)^{2}},
$$

where $R_{\max }$ and $R_{0}$ are the maximum and the initial contact diameters between the droplet and substrate, respectively. $a$ is the pillar spacing, $h$ is the wetting height of pillars, and $b$ is the width, respectively. 


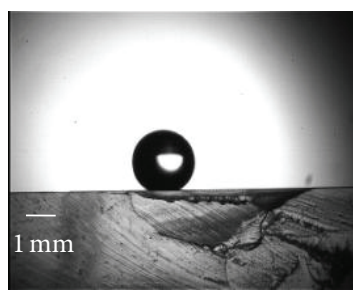

(a) $t=-10.5 \mathrm{~ms}$

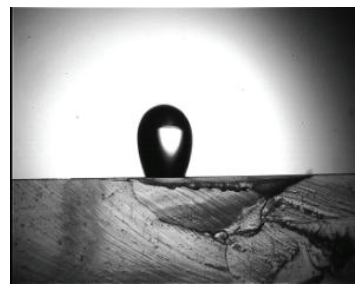

(e) $t=-3.5 \mathrm{~ms}$

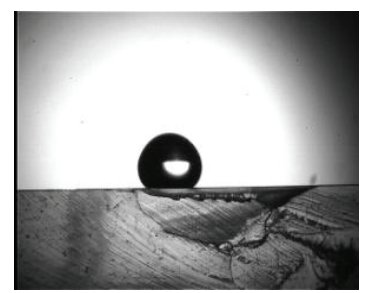

(b) $t=-9.0 \mathrm{~ms}$

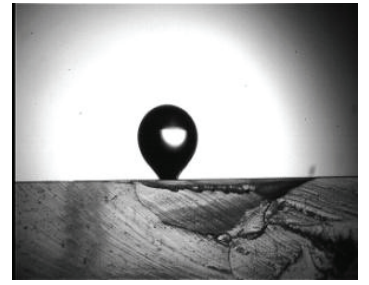

(f) $t=-1.5 \mathrm{~ms}$

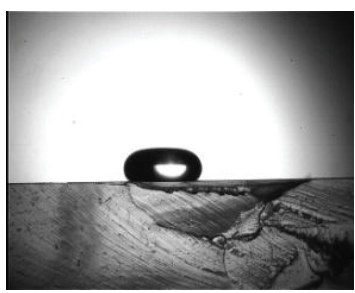

(c) $t=-7.0 \mathrm{~ms}$

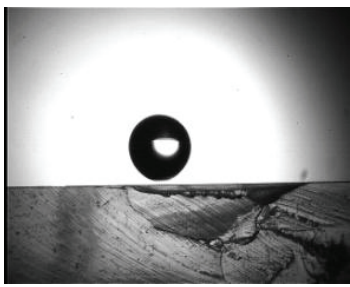

(g) $t=0 \mathrm{~ms}$

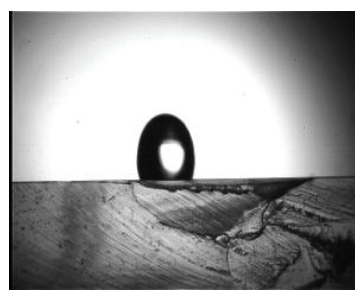

(d) $t=-4.5 \mathrm{~ms}$

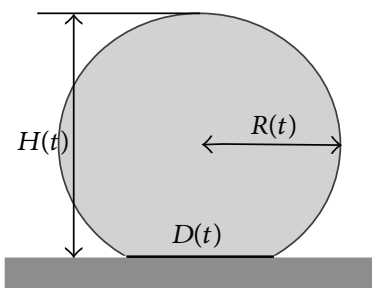

Figure 4: High speed images of the droplet bouncing-off from a vibrating plane ( $f=70 \mathrm{~Hz}, A=0.31 \mathrm{~mm}$ ). The value of $t$ for each image indicates a time relative to the moment $(t=0)$ the drop bounces off from the surface.

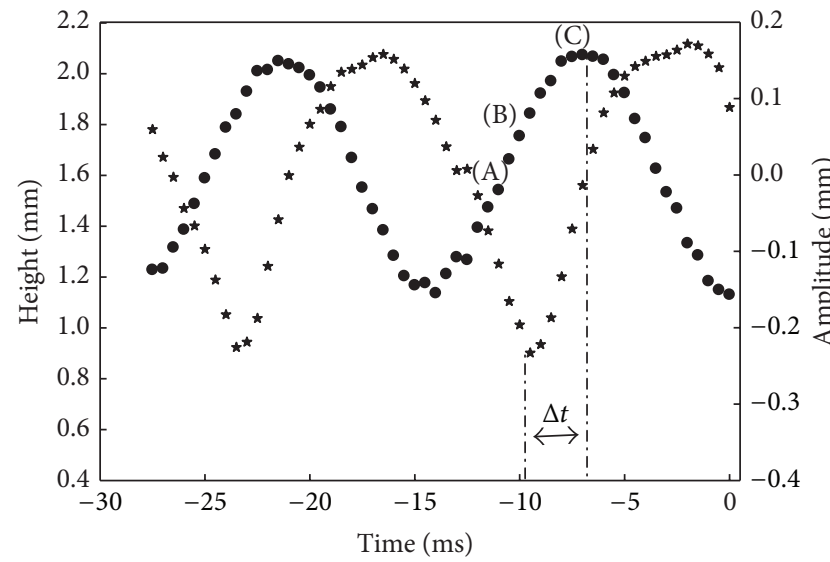

* Instantaneous height of droplet - Amplitude

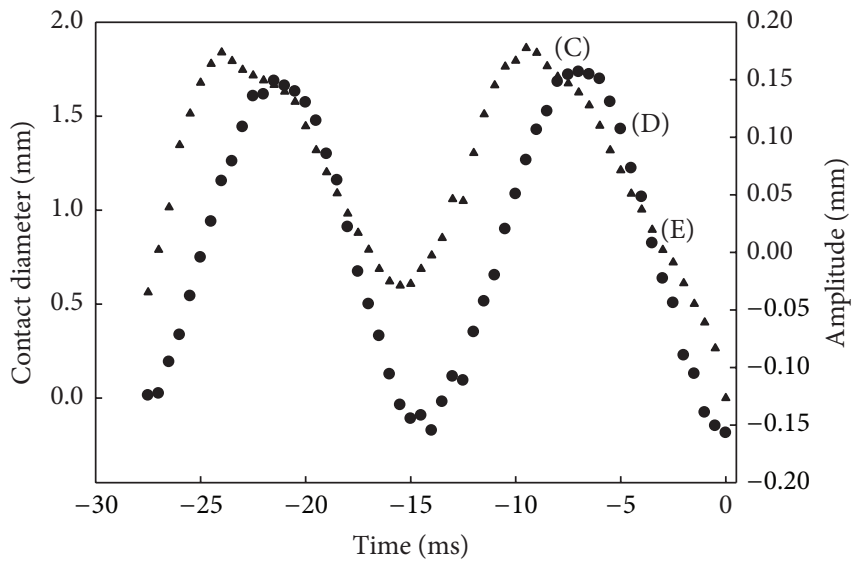

- Contact diameter

- Amplitude

(a)

(b)

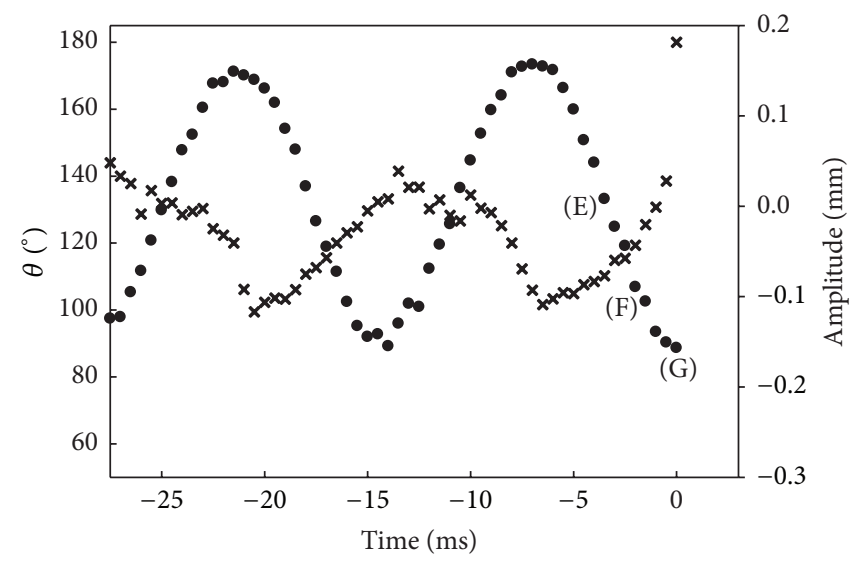

x Instantaneous contact angle $\theta$

- Amplitude

(c)

Figure 5: The transient evolution of the droplet and amplitude $A(t)$ prior to the droplet bouncing-off. (a) The droplet height $H(t)$, (b) the droplet contact diameter $D(t)$, and (c) the CA $\theta(t)$. The characteristics of the vibrated droplet on the pillared surface can generally be classified into three different categories: compression process $(A)-(C)$, restoring process $(C)-(E)$, and bouncing process $(E)-(G)$. 


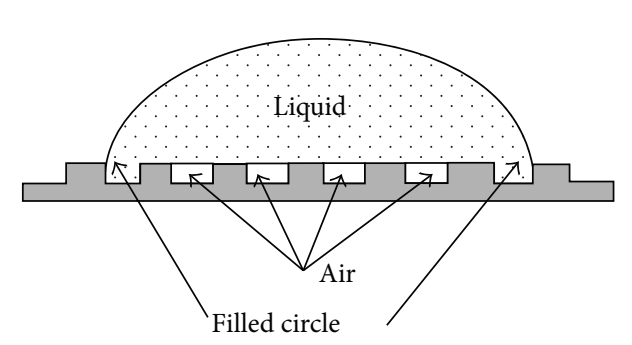

1D

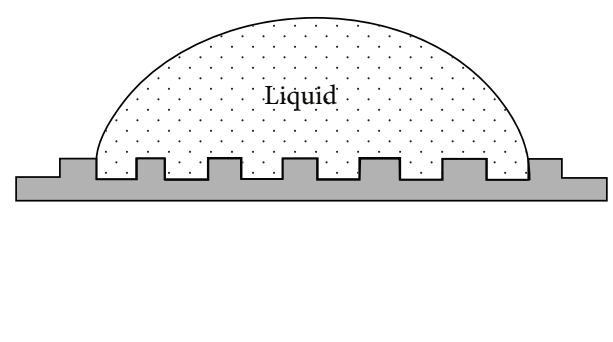

$2 \mathrm{D}$

FIgURE 6: Scheme illustrating 1D and 2D scenarios of wetting transitions [28].

The kinetic energy imparted to the drop $E_{K}$ scales as [15]

$$
E_{K} \propto \frac{1}{2} \rho V(2 \pi f A)^{2},
$$

where $\rho$ and $V$ are the density and volume of the droplet, $f$ is the external forcing frequency, $A$ is half of the peak-to-peak amplitude of vibration, and $2 \pi f A$ is the maximum speed of the speaker.

Meanwhile, the external forcing frequency $f=70 \mathrm{~Hz}$ is consistent with the resonant frequency of the droplet [33]. At resonance, the droplet picks up the kinetic energy at the highest efficiency. Assuming that the highest efficiency is $100 \%$, therefore, the kinetic energy imparted to the droplet $E_{k o}$ is

$$
E_{k o}=\frac{1}{2} \rho V(2 \pi f A)^{2} .
$$

Vibrations provide the droplet with the necessary energy for penetration into pillars:

$$
E_{k o}=E \text {. }
$$

In this paper, $2 R_{\max }=D_{\max }=1.71 \mathrm{~mm}, 2 R_{0}=D_{0}=$ $1.05 \mathrm{~mm}, A=0.31 \mathrm{~mm}, f=70 \mathrm{~Hz}, V=4 \mu \mathrm{L}, \gamma_{\mathrm{LA}}=72 \times$ $10^{-3} \mathrm{~J} / \mathrm{m}, \rho=1000 \mathrm{~kg} / \mathrm{m}^{3}$, and $\theta_{E}=113.7^{\circ}$. When substituted into (1)-(8), the wetting height $h$ can be deduced:

$$
h=-\frac{\rho V(2 \pi f A)^{2}(a+b)^{2}}{8 b \gamma_{\mathrm{LA}} \pi\left(R_{\max }^{2}-R_{0}^{2}\right) \cos \theta_{E}} \approx 27 \mu \mathrm{m} .
$$

Obviously, the droplet comes close to the Wenzel state (the height of the pillars $H=30 \mu \mathrm{m}$ ). Therefore, in the compression process, the droplet achieves a Cassie to Wenzel transition, and the transition corresponds to the $1 \mathrm{D}$ scenario of wetting transitions, which verify the analysis before.

The restoring process consists of water droplets that are considerably restored during their motion on the solid surface from the maximum positive amplitude to the equilibrium position, as shown in Figure 7(b). In the presence of the adhesion force and inertia force, the top portion of the droplet is moving towards the positive $y$-direction, but the bottom portion of the droplet is moving in the opposite direction; the droplet is stretched in the $y$-direction. Meanwhile, it results in the increasing of the liquid height and decreasing of the contact diameter between the droplet and substrate (see Figure 5), and the wetting state changed. Comparing the time points $t=-10.5 \mathrm{~ms}$ (Figure $4(\mathrm{a})$ ) and $t=-3.5 \mathrm{~ms}$ (Figure 4(e)), the contact diameter is nearly the same and the droplet is restored to Cassie state. However, because the droplet is stretched in the $y$-direction, the liquid-air line is moving and we can observe that the CAs for the two moments are very different. Namely, the CAs for the Cassie state are different and the Cassie equation is not applied to this situation. The restoring process is approximately a reversible process of the compression process, and the droplet transits from Wenzel state to Cassie state.

The bouncing process corresponds to the case where the amplitude is increased in the negative $y$-direction and the water droplet is continuously stretched as the top portion of the droplet is moving slower than the bottom portion of the droplet. In this case, the contact diameter between the droplet and substrate is decreasing until the droplet bouncing-off from the surface, as shown in Figure 7(c). Meanwhile, droplets bouncing from the superhydrophobic patterned surface only at a certain frequency and amplitude were observed. It is reasonable to suggest that only when the imparted kinetic energy is greater than the adhesion energy, the droplet can bounce from the surface.

\section{Conclusions}

The dynamic behaviors of a vibrated droplet at resonance frequency on a micropillared surface were investigated in this paper. We considered the effect of vibration on the droplet contact diameter, the CA, and the droplet height during its movement along a micropillared surface. As the amplitude of vibration increases and decreases, the dynamic behavior of a water droplet can generally be classified into three different categories: compression process, restoring process, and bouncing process. In compression process, the contact diameter between the droplet and the substrate is increasing and the liquid height and contact angle are continuously slowly decreasing. The droplet is transited from Cassie state to Wenzel state and the transition corresponds to the $1 \mathrm{D}$ scenario of wetting transitions. The restoring is an inverse process of the compression, and a Wenzel-Cassie wetting transition is observed. In the bouncing process, the contact diameter is decreasing until the droplet bouncingoff from the surface and the bouncing only happened at certain amplitude. For the micropillared surfaces, the wetting 

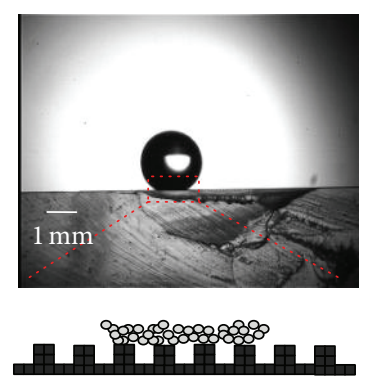

(A) $t=-10.5 \mathrm{~ms}$
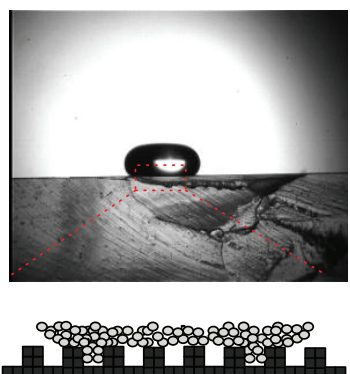

(C) $t=-7.0 \mathrm{~ms}$
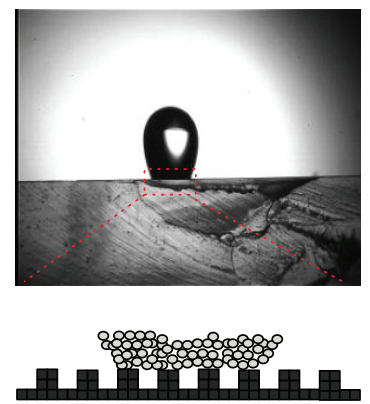

(E) $t=-3.5 \mathrm{~ms}$

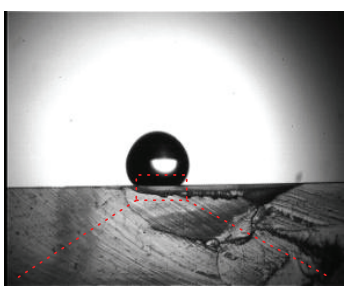

m

(B) $t=-9.0 \mathrm{~ms}$

(a) The compression process

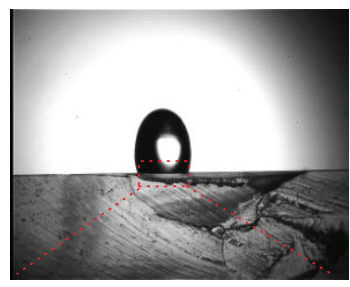

m

(D) $t=-4.5 \mathrm{~ms}$

(b) The restoring process
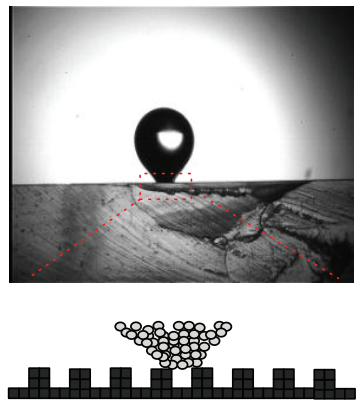

(F) $t=-1.5 \mathrm{~ms}$

c) The bouncing process

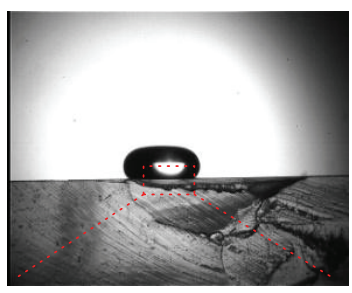

\%

(C) $t=-7.0 \mathrm{~ms}$
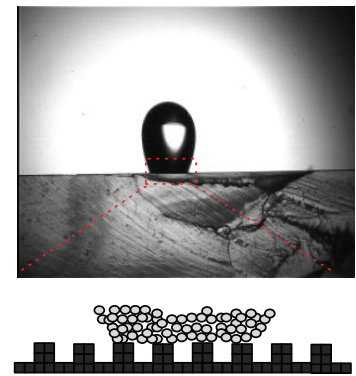

(E) $t=-3.5 \mathrm{~ms}$

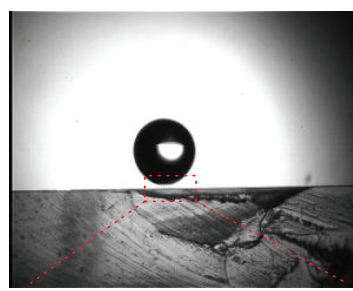

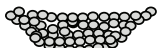

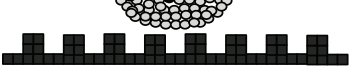

(G) $t=0 \mathrm{~ms}$

FIGURE 7: Scheme illustrating the droplet wetting behaviors.

regime could be controlled with vibration. The possibility of controlling the wetting regime with vibration may be of the utmost importance in the context of the rapid development of the applications of superhydrophobic surfaces.

\section{Conflict of Interests}

The authors declare that there is no conflict of interests regarding the publication of this paper.

\section{Acknowledgments}

The authors wish to thank the funding support from the National Natural Science Foundation of China (Grant no. 51176123), the Specialized Research Fund for the Doctoral Program of Higher Education (Grant no. 20103120120006), and Shanghai Natural Science (Grant no. 11ZR1424800).

\section{References}

[1] J. W. Rose, "Dropwise condensation theory and experiment: a review," Proceedings of the Institution of Mechanical Engineers Part A: Journal of Power and Energy, vol. 216, no. 2, pp. 115-128, 2002.

[2] T. Wu and Y. Suzuki, "Engineering superlyophobic surfaces as the microfluidic platform for droplet manipulation," Lab on a Chip, vol. 11, no. 18, pp. 3121-3129, 2011.

[3] X. Ma, J. W. Rose, D. Xu, J. Lin, and B. Wang, "Advances in dropwise condensation heat transfer: Chinese research," Chemical Engineering Journal, vol. 78, no. 2-3, pp. 87-93, 2000.

[4] D. Torresin, M. K. Tiwari, D. Del Col, and D. Poulikakos, "Flow condensation on copper-based nanotextured superhydrophobic surfaces," Langmuir, vol. 29, no. 2, pp. 840-848, 2013.

[5] Y. T. Cheng, D. E. Rodak, A. Angelopoulos, and T. Gacek, "Microscopic observations of condensation of water on lotus leaves," Applied Physics Letters, vol. 87, no. 19, Article ID 194112, 3 pages, 2005. 
[6] M. Ma and R. M. Hill, "Superhydrophobic surfaces," Current Opinion in Colloid \& Interface Science, vol. 11, no. 4, pp. 193-202, 2006.

[7] X.-M. Li, D. Reinhoudt, and M. Crego-Calama, "What do we need for a superhydrophobic surface? A review on the recent progress in the preparation of superhydrophobic surfaces," Chemical Society Reviews, vol. 36, no. 8, pp. 1350-1368, 2007.

[8] A. B. D. Cassie and S. Baxter, "Wettability of porous surfaces", Transactions of the Faraday Society, vol. 40, pp. 546-551, 1944.

[9] E. Bormashenko, "Wetting transitions on biomimetic surfaces," Philosophical Transactions of the Royal Society A: Mathematical, Physical and Engineering Sciences, vol. 368, no. 1929, pp. 46954711, 2010.

[10] A. L. Dubov, A. Mourran, M. Möller, and O. I. Vinogradova, "Contact angle hysteresis on superhydrophobic stripes," The Journal of Chemical Physics, vol. 141, no. 7, Article ID 074710, 2014.

[11] G. Whyman and E. Bormashenko, "How to make the cassie wetting state stable?” Langmuir, vol. 27, no. 13, pp. 8171-8176, 2011.

[12] Y. C. Jung and B. Bhushan, "Dynamic effects induced transition of droplets on biomimetic superhydrophobic surfaces," Langmuir, vol. 25, no. 16, pp. 9208-9218, 2009.

[13] A. M. Peters, C. Pirat, M. Sbragaglia et al., "Cassie-Baxter to Wenzel state wetting transition: scaling of the front velocity," European Physical Journal E, vol. 29, no. 4, pp. 391-397, 2009.

[14] J. B. Boreyko, C. H. Baker, C. R. Poley, and C.-H. Chen, "Wetting and dewetting transitions on hierarchical superhydrophobic surfaces," Langmuir, vol. 27, no. 12, pp. 7502-7509, 2011.

[15] J. B. Boreyko and C.-H. Chen, "Restoring superhydrophobicity of lotus leaves with vibration-induced dewetting," Physical Review Letters, vol. 103, no. 17, Article ID 174502, 2009.

[16] D. Tian, Q. Chen, F.-Q. Nie, J. Xu, Y. Song, and L. Jiang, "Patterned wettability transition by photoelectric cooperative and anisotropic wetting for liquid reprography," Advanced Materials, vol. 21, no. 37, pp. 3744-3749, 2009.

[17] Q.-S. Zheng, Y. Yu, and Z.-H. Zhao, "Effects of hydraulic pressure on the stability and transition of wetting modes of superhydrophobic surfaces," Langmuir, vol. 21, no. 26, pp. 12207-12212, 2005.

[18] P. Forsberg, F. Nikolajeff, and M. Karlsson, "Cassie-Wenzel and Wenzel-Cassie transitions on immersed superhydrophobic surfaces under hydrostatic pressure," Soft Matter, vol. 7, no. 1, pp. 104-109, 2011.

[19] E. Bormashenko, R. Pogreb, G. Whyman, Y. Bormashenko, and M. Erlich, "Vibration-induced Cassie-Wenzel wetting transition on rough surfaces," Applied Physics Letters, vol. 90, no. 20, Article ID 201917, 2007.

[20] E. Bormashenko, R. Pogreb, G. Whyman, and M. Erlich, "Resonance cassie-wenzel wetting transition for horizontally vibrated drops deposited on a rough surface," Langmuir, vol. 23, no. 24, pp. 12217-12221, 2007.

[21] N. Kumari and S. V. Garimella, "Electrowetting-induced dewetting transitions on superhydrophobic surfaces," Langmuir, vol. 27, no. 17, pp. 10342-10346, 2011.

[22] D. Mannetje, A. Banpurkar, H. Koppelman, M. H. G. Duits, D. Van Den Ende, and F. Mugele, "Electrically tunable wetting defects characterized by a simple capillary force sensor," Langmuir, vol. 29, no. 31, pp. 9944-9949, 2013.

[23] G. Liu, L. Fu, A. V. Rode, and V. S. J. Craig, "Water droplet motion control on superhydrophobic surfaces: exploiting the
Wenzel-to-Cassie transition," Langmuir, vol. 27, no. 6, pp. $2595-$ 2600, 2011.

[24] C. Dorrer and J. Rühe, "Condensation and wetting transitions on microstructured ultrahydrophobic surfaces," Langmuir, vol. 23, no. 7, pp. 3820-3824, 2007.

[25] S. L. Gras, T. Mahmud, G. Rosengarten, A. Mitchell, and K. Kalantar-Zadeh, "Intelligent control of surface hydrophobicity," ChemPhysChem, vol. 8, no. 14, pp. 2036-2050, 2007.

[26] M. Motornov, S. Minko, K.-J. Eichhorn, M. Nitschke, F. Simon, and M. Stamm, "Reversible tuning of wetting behavior of polymer surface with responsive polymer brushes," Langmuir, vol. 19, no. 19, pp. 8077-8085, 2003.

[27] Z. J. Cheng, H. Lai, N. Q. Zhang, K. N. Sun, and L. Jiang, "Magnetically induced reversible transition between cassie and wenzel states of superparamagnetic microdroplets on highly hydrophobic silicon surface," Journal of Physical Chemistry C, vol. 116, no. 35, pp. 18796-18802, 2012.

[28] E. Bormashenko, R. Pogreb, G. Whyman, and M. Erlich, "Cassie-Wenzel wetting transition in vibrating drops deposited on rough surfaces: is the dynamic Cassie-Wenzel wetting transition a 2D or 1D affair?," Langmuir, vol. 23, no. 12, pp. 65016503, 2007.

[29] V. Vandaele, A. Delchambre, and P. Lambert, "Acoustic wave levitation: handling of components," Journal of Applied Physics, vol. 109, no. 12, Article ID 124901, 2011.

[30] S. Mettu and M. K. Chaudhury, "Vibration spectroscopy of a sessile drop and its contact line," Langmuir, vol. 28, no. 39, pp. 14100-14106, 2012.

[31] X. Noblin, A. Buguin, and F. Brochard-Wyart, "Vibrated sessile drops: transition between pinned and mobile contact line oscillations," European Physical Journal E, vol. 14, no. 4, pp. 395404, 2004.

[32] X. Zhang, F. Shi, J. Niu, Y. Jiang, and Z. Wang, "Superhydrophobic surfaces: from structural control to functional application," Journal of Materials Chemistry, vol. 18, no. 6, pp. 621-633, 2008.

[33] W. Lei, Z.-H. Jia, J.-C. He, and T.-M. Cai, "Dynamic properties of vibrated drops on a superhydrophobic patterned surface," Applied Thermal Engineering, vol. 62, no. 2, pp. 507-512, 2014.

[34] E. Bormashenko, A. Musin, G. Whyman, and M. Zinigrad, "Wetting transitions and depinning of the triple line," Langmuir, vol. 28, no. 7, pp. 3460-3464, 2012. 

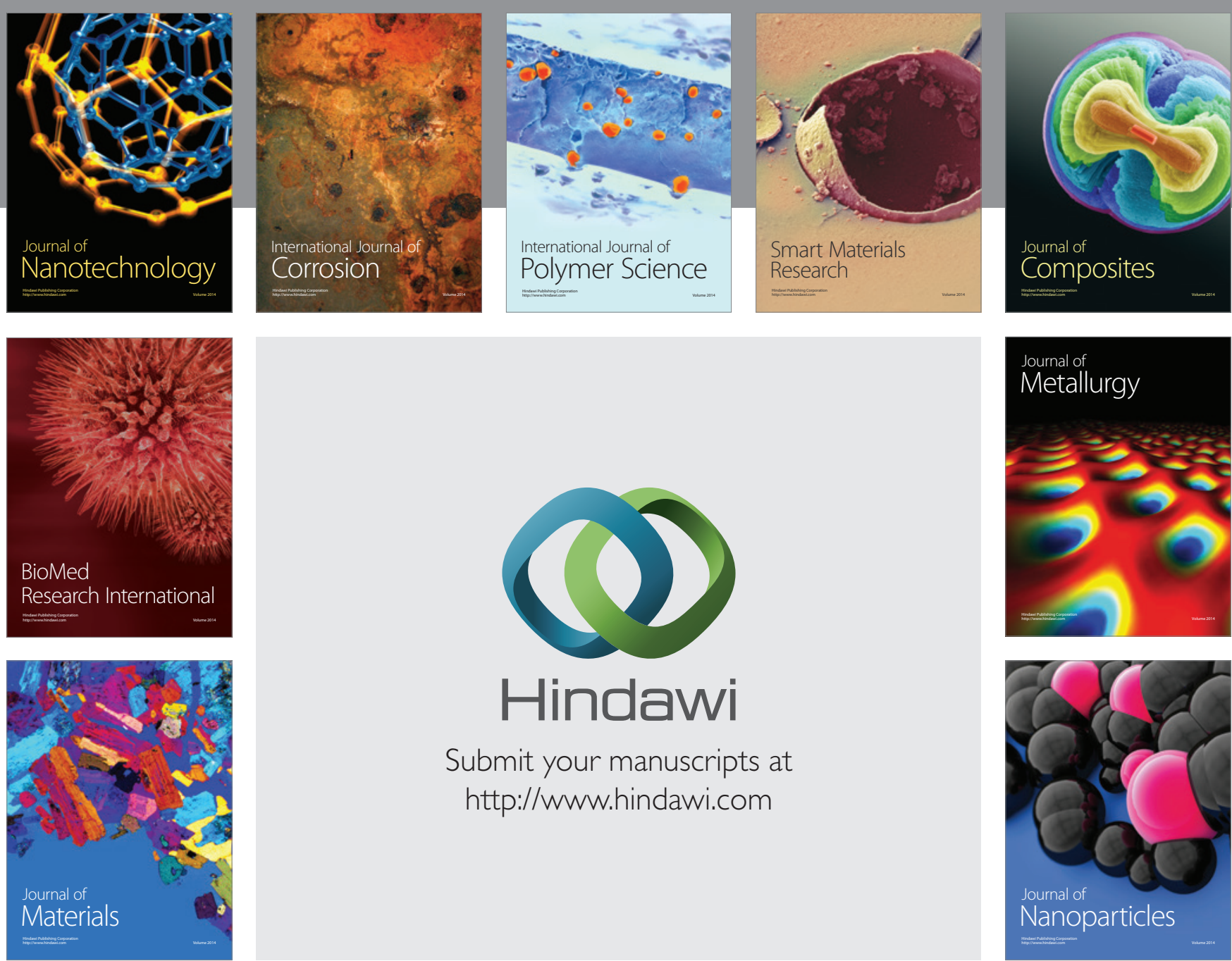

\section{Hindawi}

Submit your manuscripts at

http://www.hindawi.com

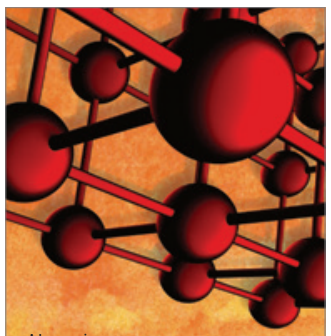

Materials Science and Engineering
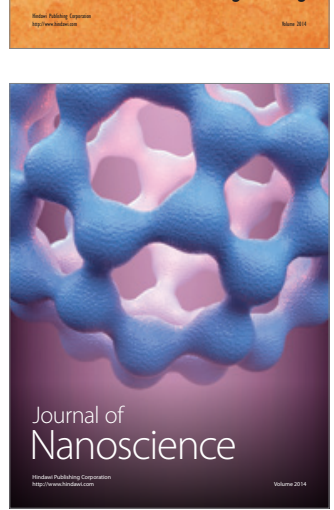
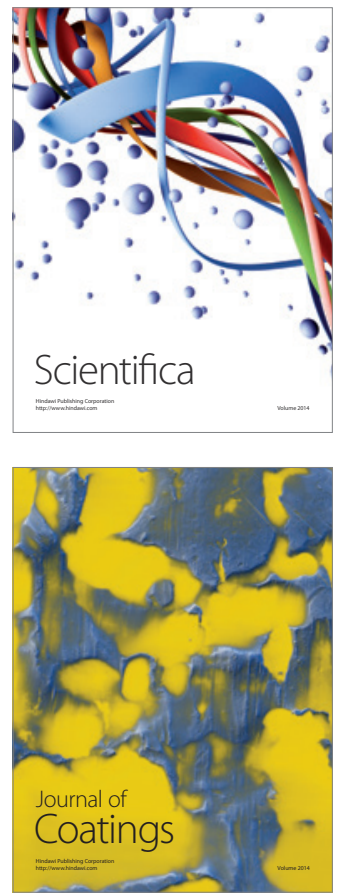
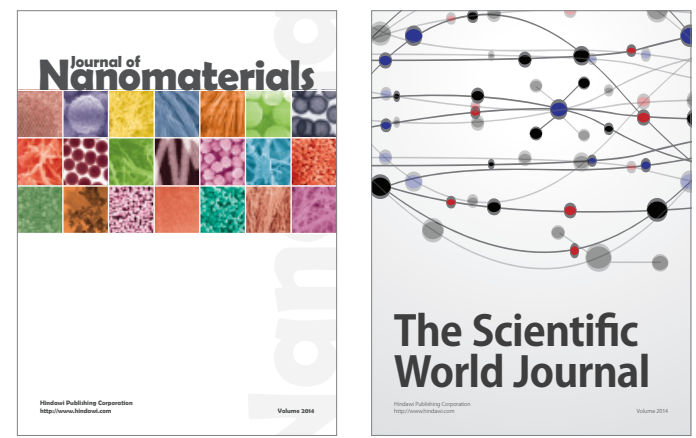

The Scientific World Journal
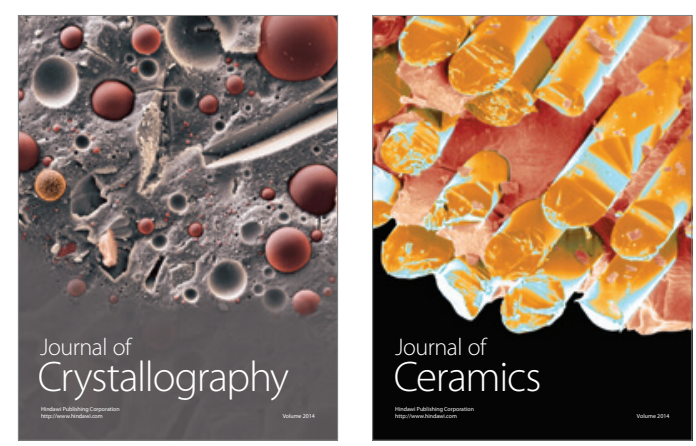
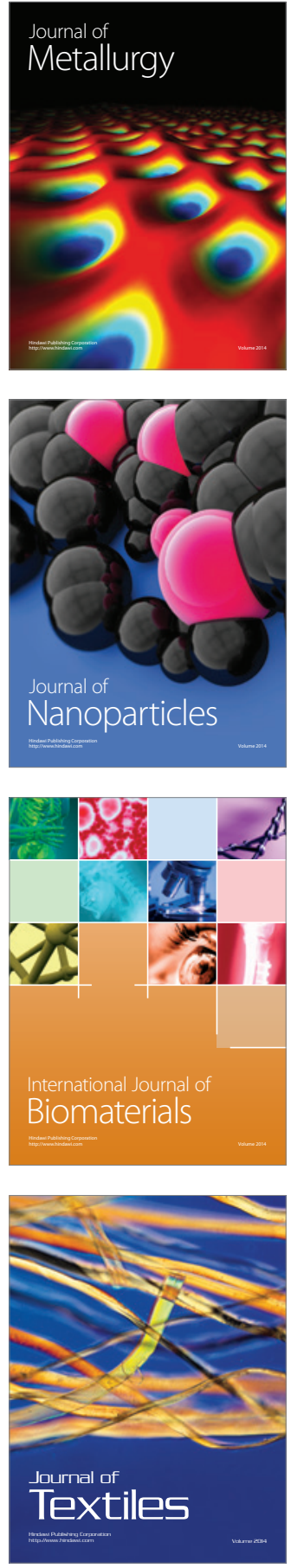